\title{
SUR LES DERMATOPHYTES
}

\section{QUI CAUSENT LA TEIGNE FAVEUSE HUMAINE}

\author{
Par M. LANGERON et M. BAEZA
}

Ce travail est basé sur l'étude systématique de 304 cas de favus provenant du Nord de l'Afrique (zone de protectorat espagnol au Maroc). Pour chaque cas, il a été fait un examen microscopique du poil et des cultures qui ont été suivies au moins pendant 6 mois, avec 3 repiquages au moins pour chacune, en divers milieux. Cela nous a permis d'isoler six types de champignons que nous considérons comme les agents de cette maladie. Dans une note statistique préliminaire, l'un de nous (Baeza 1934) leur donne provisoirement le nom d'Achorion schönleini. Cependant, les cultures de ces parasites présentent de telles différences qu'il est nécessaire d'admettre que le favus est produit par des types distincts de champignons et que, dans le genre Achorion, il existe plusieurs espèces.

En précisant ces différences et en groupant les formes affines, nous pouvons établir, parmi les agents de la teigne faveuse que nous avons étudiés, trois grands groupes :

A. - Champignons à culture glabre, spongieuse, irrégulière, se développant à la surface du milieu (milieu de Sabouraud), sans aucunement y pénétrer, au moins en apparence. Ce premier groupe est constitué par l'Achorion schönleini classique, obtenu 175 fois.

B. - Champignons qui s'insèrent sur le milieu de culture, sans donner la sensation de simple contact comme pour les précédents, mais plutôt d'adhérence ; leur croissance est surtout superficielle. Ils présentent quatre types que nous décrirons plus loin :

$\begin{array}{ccc}\text { type pittalugai } & \text { rencontré dans } & 15 \text { cas ; } \\ \text { - talicei } & - & 9- \\ - \text { debueni } & - & 22- \\ - \text { brumpti } & - & 6-\end{array}$

C. - Champignons qui croissent de préférence dans la profondeur du milieu, à l'intérieur duquel ils pénètrent sous forme rayonnée et régulière; le développement superficiel est faible. Tous les champignons de ce groupe peuvent se ramener à un seul type :

type milochevitchi rencontré dans 77 cas.

Annales de Parasitologie, T. XI, No $4,-1^{\text {er }}$ juillet 1936 , p. 385-402.

25 . 
ETUde SPÉciale des NOUVEAUX TYPES

$1^{\text {er }}$ GROUPE, - COLONIES SPONGIEUSES, SUPERFICIELLES

\section{Type Achorion schönleini ( $\mathrm{pl}, \mathrm{XV}$ )}

Caractères cliniques. - Favus typique, 148 cas ; un cas de favus à très petits godets; deux cas de favus sans godets ; favus résiduel, 12 cas ; favus ichthyoïde, 8 cas ; favus suppuré, 4 cas. En tout, 175 cas.

Examen microscopigue du poil (dans le chlorallactophénol). Aspect typique de l'Achorion schönleini (fig. 1 et 3, pl. XV).

Culture première. - Sur milieu d'épreuve glycosé, début en 3-6 jours. Colonies glabres, membraneuses, creuses, cireuses, adhérentes au milieu, non pénétrantes, spongieuses, très proéminentes et, en certains cas, légèrement farineuses à la surface (fig. $4, \mathrm{pl}$ XV).

Cultures secondes. - Sur milieu d'épreuve glycosé, ou maltosé, et sur milieu polysucré de Baeza (1). Mêmes caractères que pour les cultures premières.

Sur milieu de conservation de Sabouraud : colonies spongieuses, absolument glabres.

Sur grains d'orge: colonies blanches, plâtreuses ou farineuses, pauvres.

Sur milieu de Löwenstein : colonies rugueuses, brunes, peu proéminentes, adhérentes au milieu et glıbres.

Morphologie microscopique (fig. 6, pl. XV). - En culture sur lames gélosées sur milieu polysucré de Baeza, suivant la méthode de Rivalier modifiée par Langeron et Guerra (2) : hyphes cloisonnées polymorphes, organes nodulaires $(\mathrm{N})$ abondants, chlamydospores intercalaires, terminales (C) ou en chaînes, ayant l'aspect d'arthrospores (E) : chandeliers faviques (F) et aleuries (A) peu abondantes, mais typiques, portées par une portion spécialisée du mycélium.

(1) Le milieu polysucré de Baeza a la composition suivante :

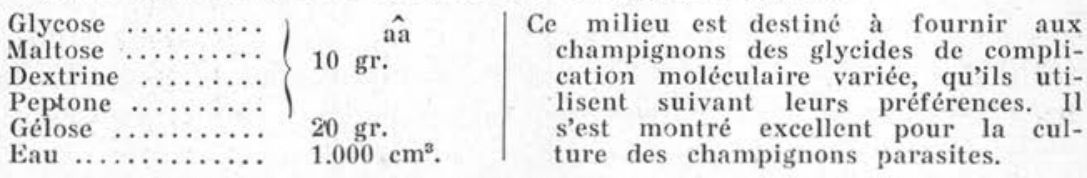

(2) Langeron (M.). - Précis de Microscopie, 5e édit., 1934, Paris, Masson et $\mathrm{C}^{10}$; ef. p. 1122-1124. 
Dans les cultures en tubes sur les divers milieux sucrés, on trouve les mêmes éléments, mais à un état plus imparfait.

En tubes, sur milieu de conservation et milieu de Löwenstein, on ne trouve que des chlamydospores, isolées ou en chaînes et des chandeliers faviques.

Sur grains de céréales, on trouve une quantité de petites spores détachées qui sont très probablement des aleuries.

$2^{\circ}$ GROUPE. - COLONIES NON SPONGIEUSES, ADHERENTES

\section{Type pittalugai (1) (pl. XV et XVI)}

Caractères cliniques. - Dix cas de favus commun, un cas de favus suppuré, un cas de favus ichthyoïde et trois cas de favus résiduel. En tout quinze cas.

Examen microscopigue du poil. - Aspect du favus commun. (fig. 5, pl. XV).

Culture première. - Sur milieu épreuve glycosé, début en 3 ou 5 jours. Colonies arrondies, superficielles, croissant en forme d'assiette ou de bassinet, avec un rebord saillant quelquefois double (fig. 2, pl. XV). La surface des colonies est couverte de très petites crevasses ou stries qui lui donnent un aspect de peau de tortue ; quelquefois cette surface est très légèrement plâtreuse.

Cultures Secondes. - Sur les divers milieux sucrés, on retrouve les caractères de la culture première.

Sur milieu de conservation, colonies absolument glabres, ayant la morphologie classique de l'Achorion commun.

Sur milieu de Löwenstein, colonies absolument glabres, régulièrement plissées et de couleur brune.

Sur grains d'orge, colonies pauvres, blanches, duveteuses et farineuses.

Morphologie microscopiQue (fig. 8, pl. XVI). - En cultures sur lames en milieu polysucré : mycélium cloisonné avec chlamydospores (C) intercalaires et terminales. Chaînes de petites chlamydospores portant des éléments latéraux dont l'assemblage ressemble tantôt à des arthrospores, tantôt à une véritable fructification. Nombreux organes nodulaires $(\mathrm{N})$ avec hypertrophies, paraissant servir

(1) Dédié au Pnof. G. Pittaluga (Faculté de Médecine de Madrid). 
d'ornements. Quelques rameaux rachitiques d'aleuries (A). Quelques chlamydospores latérales, groupées par deux ou par trois, rappelant la forme d'un fuseau (ch.). Organes pectinés (P) et chandeliers faviques $(\mathrm{F})$.

En tubes, sur les divers milieux sucrés, mêmes caractères, mais plus difficiles à reconnaître.

En tubes sur milieux de conservation et de Löwenstein, mycélium fragile fragmenté, chlamydospores nombreuses et libres.

Sur grains de céréales, nombreuses spores libres de différente taille, probablement chlamydospores et aleuries. Nous n'avons jamais vu ni vrai fuseau ni spirale.

\section{Type brumpti (1) (pl. XVI et XVII)}

Caractères cliniques. - Favus typique, cinq cas ; favus résiduel, un cas. En tout, six cas.

EXamen microscopique du poil. - Aspect du favus commun (fig. 11, pl. XVI).

Culture première. - Sur milieu d'épreuve glycosé, début en cinq ou six jours. Colonies absolument glabres, un peu jaunâtres et granuleuses, comme si elles étaient formées de pâte de semoule, superficielles, non pénétrantes, recouvertes au bout de cinq mois d'un duvet de type pléomorphique (fig. 12 , pl. XVI).

Cultures secondes (fig. 13, pl. XVI). - Sur milieu de conservation de Sabouraud, colonies rugueuses mais absolument glabres, dont l'aspect rappelle de loin celui des colonies de Trichophyton cerebriforme.

Sur milieu de Löwenstein : colonies absolument glabres, rugueuses, couleur terre de Sienne.

Sur grains de céréales : colonies blanches, pauvres, plâtreuses, sur lesquelles apparait rapidement un duvet stérile d'aspect pléomorphique.

Morphologie microscopiQue (fig. 9, pl. XVI). - En culture sur lames sur milieu polysucré : mycelium cloisonné polymorphe avec nombreux organes nodulaires $(\mathrm{N})$. Nombreux rameaux portant des aleuries parfaites, fréquemment insérés au voisinage des organes nodulaires et qui, par leur différenciation spéciale, acquièrent la signification de véritables aleuriophores. Chlamydospores (C) en

(1). Dédié au Prof. E. Brumpt, Directeur de l'Institut de parasitologie de la Faculté de médecine de Paris. 
chaînes fragiles, du type arthrospores. Quelques chlamydospores intercalaires ou terminales. Ni chandeliers faviques ni fuseaux.

Dans les cultures secondes en tubes, sur les divers milieux sucrés, on retrouve les mêmes éléments, mais avec difficulté.

Dans les cultures secondes, sur milieu de conservation et milieu de Löwenstein, de même que dans les cultures premières en tubes sur milieu d'épreuve glycosé, on ne trouve que des chlamydospores isolées ou en nombreuses chaînes fragiles et quelques chandeliers faviques.

Nous n'avons jamais vu ni spirales, ni fuseaux.

\section{Type debueni (1) (pl. XVII et XVIII)}

Caractères cliniques. - Favus commun, 16 cas ; favus sans godets, 4 cas ; un cas de favus à très petits godets et un cas de favus ichthyoïde. En tout, 22 cas.

Examen microscopique du poll. - Aspect du favus commun (fig. 22, pl. XVIII).

Culture première. - Sur milieu d'épreuve glycosé, début en 7 à 8 jours. Petites colonies à développement très lent, peu rugueuses et peu pénétrantes, qui se couvrent en vieillissant d'une poussière farineuse.

Cultures secondes. - Sur milieux sucrés divers, mêmes caractères que la culture première.

Sur milieu de conservation, colonies absolument glabres et très petites.

Sur milieu de Löwenstein, colonies glabres, rugueuses, aplaties, de couleur un peu brune.

Sur grains de céréales, colonies très pauvres, blanches, plâtreuses.

Morphologie microscopiQue (fig. 16, pl. XVII). - En cultures sur lames sur milieu polysucré : mycélium cloisonné avec nombreux organes nodulaires $(\mathrm{N})$. Chlamydospores intercalaires et terminales (C). Chandeliers faviques (F) à l'extrémité desquels se condense le protoplasma, ce qui donne naissance à des spores qui aussitôt se détachent. Chaînes de chlamydospores (E) ayant l'apparence d'arthrospores. Aleuries (A) rares; par exception, on a pu voir un rameau compliqué (fig. 17, pl. XVII).

(1) Dédié au $\mathrm{D}^{\mathrm{r}}$ Sadi de Buen, Inspecteur général des Institutions sanitaires d'Espagne. 
Il y a quelques rares éléments ayant l'aspect de fuseaux, mais que nous ne pouvons considérer comme tels, ce sont plutôt des modalités de chlamydospores.

Dans les tubes des divers milieux sucrés, on observe les mêmes éléments, mais avec plus de difficulté et moins de précision.

Sur milieux de conservation et de Löwenstein, on ne voit que des chaînes fragiles de chlamydospores et une quantité de chlamydospores libres de toutes tailles.

Sur grains de céréales, les aleuries sont plus nombreuses, mais leur étude est difficile, parce qu'elles se détachent très facilement. Il y a aussi beaucoup de chlamydospores isolées ou en chaînes. Nous n'avons jamais vu de spirales.

\section{Type talicei (1) (pl. XVIII)}

Caractères cliniques. - Sept cas de favus commun, un cas de favus suppuré et un cas de favus sans godets. En tout, neuf cas.

Examen microscopigue du poil. - Aspect d'Achorion (fig. 23, pl. XVIII).

Culture première. - Sur milieu d'épreuve glycosé, début en 7-9 jours. Colonies légèrement pénétrantes et légèrement veloutées, pulvérulentes ; accroissement circulaire ; bourrelet extérieur proéminent, réuni au centre par des rayons plus ou moins irréguliers. En vieillissant, les colonies se fendillent et, par éversion, montrent la surface interne jaune cire de la membrane qui les constitue.

Cultures secondes. - Sur les divers milieux sucrés, mêmes caractères que les cultures premières.

Sur milieu de conservation, colonies irrégulières, spongieuses, absolument glabres.

Sur grains de céréales, colonies blanches farineuses, très rachitiques.

Morphologie microscopique (fig. 24, pl. XVIII). - En culture sur lames sur milieu polysucré : mycélium cloisonné polymorphe. Organes nodulaires (N). Aleuries (A) peu abondantes. Chaînes de chlamydospores (E). Chlamydospores (C) intercalaires et terminales. $\mathrm{Ni}$ chandeliers faviques, ni spirales.

Dans les cultures en tubes, sur divers milieux sucrés, on trouve les mêmes éléments avec difficulté.

(1) Dédié au Prof. R.-V. Talice (Faculté de Médecine de Montevideo). 
Dans les cultures en tubes, sur milieux de conservation et de Löwenstein, on peut voir des chlamydospores isolées ou en chaînes.

Dans les cultures sur grains de céréales, on trouve une grande quantité de spores de différentes tailles (aleuries et chlamydospores), mais presque toujours détachées.

\section{3e GROUPE. - COLONIES PLUS OU MOINS}

\section{COMPLÉTEMENT IMMERGÉES}

\section{Type milochevitchi (1) (pl. XIX)}

Caractères cliniques. - Favus commun, 56 cas ; favus suppuré, 6 cas ; favus résiduel de l'adulte, 8 cas ; favus sans godets, 3 cas ; favus ichthyoïde, 4 cas. En tout, 77 cas.

Ce type paraît assez répandu en Asie, car on l'a trouvé, comme nous le verrons plus loin, à Formose, à Pékin et dans l'Inde.

Examen microscopigue du poil. - Aspect de favus commun, même dans les cas d'infection résiduelle légère (fig. 28 , pl. XIX).

Culture première. - Sur milịeu d'épreuve glycosé, début en 3-4 jours. Colonies se développant en majeure partie dans la profondeur du milieu, dans lequel elles pénètrent sous la forme rayonnante et régulièrement grumeleuse. Seule émerge la partie centrale de la colonie qui apparaît comme une hernie à surface rugueuse irrégulière, demi-glabre, demi-plâtreuse. Lorsque la colonie vieillit, certaines parties des rayons profonds donnent à la surface un début de colonie punctiforme qui suit la même évolution que la hernie centrale, mais sans jamais acquérir sa taille, sans doute parce que la sécheresse du milieu ne le permet pas (fig. 27, pl. XIX).

Cultures secondes. - Sur les divers milieux sucrés, mêmes caractères que les cultures premières.

Sur milieu de conservation, colonies absolument glabres, pénétrant dans le milieu, mais pas autant que les précédentes et, en outre, la pénétration n'est pas aussi nettement rayonnante, mais en masse compacte, irrégulière.

Sur milieu de Löwenstein, colonies glabres, aplaties, brunes, pénétrant peu dans le milieu où elles ne forment pas de rayons.

Sur grains de céréales, colonies légèrement veloutées, plâtreuses, de développement pauvre, mais pénétrant dans le grain.

(1) Dédié au $\mathrm{D}^{\mathrm{r}} \mathrm{S}$. Milochevitch (Institut central d'hygiène de Belgrade). 
Morphologie microscopiQue (fig. 30, pl. XIX). - En culture sur lames sur milieu polysucré : mycélium cloisonné polymorphe, avec nombreuses anastomoses et organes nodulaires (N). Aleuries (A) abondantes, en grappe simple (Acladium) le plus souvent et dans quelques cas portées par un sporophore à ramification rudimentaire. Chlamydospores (C) intercalaires et terminales ou en chaînes (E). Chandeliers faviques (F) présentant quelquefois une condensation protoplasmique apicale qui finit par donner lieu à la formation de petites spores ( $f e$ ) détachées ou en grappe, et dont nous n'avons pu établir l'identité avec les aleuries. On peut observer les mêmes éléments, quoique d'une manière plus imparfaite, sur milieux d'épreuve glycosé, maltosé et polysucré.

Sur milieu de conservation et milieu de Löwenstein, on ne voit que des chaînes fragiles de chlamydospores ressemblant à des arthrospores, avec de nombreuses chlamydospores intercalaires et terminales.

Sur grains de céréales, il y a beaucoup d'aleuries détachées et de chlamydospores isolées ou en chaînettes.

\section{Signification de ces ÉlÉments morphologiQues}

Le mycelium cloisonné existe dans tous les types, mais le mode de cloisonnement est variable : les cloisons sont d'autant plus rapprochées que la culture est plus glabre et d'autant plus éloignées qu'elle est plus duveteuse.

Les chandeliers faviques sont une modalité du mycelium due à la non-adaptation du champignon au milieu ; ce sont des formes de souffrance (Langeron et Milochevitch) qui disparaissent quand le milieu se trouve favorable.

Les chlamydospores en chaine sont des organes de dissémination formés par condensation protoplasmique à l'intérieur de la membrane du thalle; cette dernière est toujours facile à distinguer parce qu'elle se colore mal avec les réactifs communs, qui, au contraire, colorent parfaitement ces chlamydospores. Malgré leur disposition en chaine et leur aspect, elles ne peuvent être considérées comme de vraies arthrospores. Puisque ces éléments ne sont pas des arthrospores, ces champignons, outre d'autres raisons, ne peuvent être rangés dans le groupe des arthrosporés.

Les chlamydospores isolées ont la signification générale qui leur a été donnée : accumulation ou condensation de protoplasma organisée en formes de résistance. Nous n'avons pas trouvé de différences à ce point de vue entre les divers types de champignons que nous avons étudiés. 
Les formes en fuseau que nous avons rencontrées ne peuvent être comparées aux fuseaux parfaits, tels, par exemple, que ceux du Sabouraudites gypseus. Ce sont des formes avortées de véritables fuseaux ou des chaînes formées de grosses chlamydospores ayant pris l'aspect fusiforme.

Les aleuries que nous avons rencontrées sont absolument parfaites et typiques : ce sont de vrais organes de dissémination, au même titre que les conidies des Penicillium. Leur insertion se fait, non en un point quelconque du thalle, mais sur une portion qui ne paraît pas destinée à autre chose qu'à produire et à porter les aleuries ; par suite, elle prend la signification d'un véritable sporophore, dans le cas particulier d'un aleuriophore. Ce caractère d'insertion sur un organe différencié, d'où ne partent pas des filaments stériles, les distingue des spores latérales qui se forment sur les hyphes communes et qui, en certains cas, peuvent simuler des rameaux aleuriophores du type Acladium. Les aleuries sont constantes dans les types de champignons faviformes humains que nous avons étudiés, quand on les cultive sur un milieu approprié. Ces champignons peuvent ne pas présenter d'aleuries quand on les cultive sur d'autres milieux non favorables. On ne peut considérer ces spores comme des organes de dégénérescence ou pléomorphiques, comme le veut Grigoraki, puisqu'elles se forment dans des souches jeunes et qu'elles apparaissent en 8 jours dans les cultures sur lames.

Nous ne sommes pas les premiers à avoir vu des aleuries dans les Achorion humains. Sabouraud et Negroni (1929) les ont observées, quoiqu'imparfaites, dans la souche de Payenneville. Catanei (1930 et 1931) les a vues dans l'Achorion schönleini cultivé sur grains de blé et sur carotte. Sur ce dernier milieu il a vu des appareils sporifères simples du type Acladium. Grigorakis (1933) les a vues aussi, mais il les considère comme des éléments pléomorphisés pouvant être produits par tous les dermatophytes. Nous ne pouvons que confirmer ces constatations et les généraliser en disạnt que, dans les 304 souches que nous avons étudiées, la présence d'aleuries plus ou moins parfaites a été constante et que leur production est en raison inverse de celle des chlamydospores isolées ou en chaines. Ces éléments sont sûrement des organes de dissémination et le champignon forme l'un ou l'autre suivant le milieu sur lequel il vit. Sur les milieux peptonés et albuminoïdes, on ne voit que des chlamydospores ; sur les milieux sucrés et solides, ce sont les aleuries qui apparaissent avec le plus de facilité. Des phénomènes semblables ont été observés par nous chez les Penicillium et les Hormodendron (Baeza 1934). 
Les organes nodulaires sont des lieux de convergence et d'anastomose d'hyphes de diverses provenances, dans lesquelles se forment de grandes condensations protoplasmiques (spores ?) desquelles partent des filaments courts et déliés qui forment une sorte de chevelure ou d'ornement autour de la masse centrale. Il est possible qu'au niveau de ces organes nodulaires, il y ait une fusion de protoplasmes et de noyaux, et que ces formations ne soient dues ni à un accident, ni à la résistance du milieu, mais bien à une attraction mutuelle ou peut-être à la nécessité d'une fusion d'hyphes qui paraissent se conjuguer à ce niveau.

Voici l'énumération des types d'Achorion, distincts du type classique, qui ont été rencontrés par d'autres auteurs dans des conditions semblables à celles que nous venons de décrire :

Hashimoto, Ishibashi, Iwatake et Ota (1927) ont trouvé, en Mongolie, un champignon qu'ils ont nommé A. schönleini, var. mongolica dont la description rappelle un peu celle de notre type milochevitchi (pl. XIX).

Hashegawa (1927) a isolé à Formose, 18 fois sur 27 cas, un Achorion, auquel il a donné le nom d'A. formoseum. Le cheveu, terne et peu cassant, renfermait des filaments et des chaînettes longitudinales de spores. Les colonies se rapprochent singulièrement de notre type milochevitchi : d'abord jaune clair et humides, elles brunissent vers le $15^{\circ}$ jour ; leur partie aérienne est plissée et elle est entourée de prolongements radiés, immergés dans le milieu de culture. Un duvet blanc est apparu vers le second mois.

L'examen microscopique de ces colonies a montré un mycelium cloisonné et ramifié, des chlamydospores intercalaires, pédiculées ou en chaînes, des chandeliers faviques, des clous faviques (corps jaunes) et des organes nodulaires.

C'est probablement ce même champignon qui a été vu en Chine par K. In (1929), dans la basse vallée du Yang-Tsé-Kiang, et par Chen, Kurotchkin et $\mathrm{Hu}$ (1931), à Pékin. Ces derniers auteurs ont retrouvé dans leurs types III et IV les files de spores dans les cheveux et les colonies entourées de rayons immergés ; leur type I ressemble à notre type debueni et leur type II à notre type brumpti.

Dey et Maplestone (1936) ont fait connaître, dans l'Inde anglaise, un nouveau type d'Achorion (A. actoni n. sp.), isolé dans un seul cas. Les colonies glabres, faviformes, de couleur brun jaunâtre, sont couvertes au début d'une poussière crayeuse qui disparaît au bout de 3 ou 4 semaines. Il y a sécrétion d'un pigment brun qui diffuse dans le milieu. Par son halo formé de filaments submergés, ce champignon se rattache à notre type milochevitchi. 
L. Grigoraki (1933) a décrit un champignon qu'il a isolé de lésions circinées, squameuses, un peu érythémateuses, du tronc et des membres, accompagnées de deux petits godets sur la nuque, chez un soldat malgache. Il a donné à ce champignon le nom d'Arthrosporia gougeroti. Les colonies sont glabres, sillonnées, spongieuses et ressemblent à celles de l'Achorion schönleini, mais elles en diffèrent par l'apparition, au bout d'un certain temps, d'un duvet formé de filaments grêles, portant des aleuries (voir plus haut, p. 393).

Des photographies d'A. schönleini, qui s'éloignent du type classique et qui ressemblent plus ou moins à ceux que nous décrivons ici, se trouvent dans des publications de divers auteurs et notamment dans un mémoire de Catanei (1933).

Enfin, dans la Mycothèque du Laboratoire de parasitologie de la Faculté de médecine de Paris, les souches $\mathrm{n}^{\circ 5} 376,529$ et 706 , pl. XX, fig. 33, diffèrent, par leur morphologie macroscopique sur milieux d'épreuve de Sabouraud et sur milieu polysueré, des souches d'A. schönleini typiques, bien qu'elles soient d'origine humaine. Chose curieuse, deux de ces souches (529 et 376) sont d'origine africaine (529, D Catanei, El Kantara, Algérie ; 376, D ${ }^{r}$ Smith, Lagos, Nigeria).

\section{SPÉCIFICITÉ}

La découverte de ces divers types de champignons comme agents du favus pose le problème de la spécificité de cette lésion et des parasites qui la produisent.

L'équation établie par Sabouraud : favus $=$ Achorion, a été détruite par l'étude complète des champignons nommés par Sabouraud Achorion gypseum, A. quinckeanum et A. gallinx. En effet, ces champignons sont très éloignés botaniquement et complètement différents de l'Achorion schönleini; ils se rapprochent beaucoup plus du groupe de champignons que Sabouraud a nommés Microsporum (comme l'ont pleinement démontré Langeron et Milochevitch, 1930) pour indiquer qu'ils sont la cause des microspories humaines. On ne peut donc plus dire : favus = Achorion, puisque le favus du rat et celui de la poule sont produits par des champignons à morphologie de Microsporum, placés par Langeron et Milochevitch dans le genre Sabouraudites.

On ne peut pas dire non plus : favus humain $=$ Achorion, puisque ce favus peut être produit par le Sabouraudites gypseus (Bodin) et par $S$. quinckeanus (anciens Achorion).

En outre, un cas clinique de favus produit par un Trichophyton faviforme mégasporé ( $T$. album) a été décrit par Milochevitch (1933). 
Bruno-Bloch (1911) a signalé aussi un cas de godets faviques produits par le Trichophyton violaceum qu'il nomme dans ce cas Achorion violaceum. Enfin, l'un de nous a observé aussi des godets produits par $T$. violaceum ; toutefois, nous devons reconnaitre que ces godets étaient cliniquement et microscopiquement distincts de ceux du favus classique.

L'Achorion forme, dans la classification de Langeron et Milochevitch (1930), un genre qui comprend une seule espèce (A. schönleini). Dans toutes les classifications, on ne donne pour ce champignon que des caractères négatifs : colonies non duveteuses, sans fuseaux, sans aleuries... et si ces éléments se rencontrent en quelque cas, ils sont si isolés et si imparfaits qu'on peut les considérer comme un fait exceptionnel.

Pourtant, au cours de nos recherches, dans nos 304 souches isolées d'autant de cas de favus indubitable, nous avons rencontré des champignons dont les caractères morphologiques ne sont pas aussi négatifs que ceux de l'A. schönleini et qui, au point de vue botanique, les rapprochent singulièrement des Trichophyton faviformes d'origine animale, produisant certaines trichophyties suppurées du type endo-ectothrix mégasporé.

Les Achorion humains que nous venons de décrire, d'une part, les Trichophyton mégasporés faviformes d'origine animale, d'autre part, constituent pour nous un groupement homogène qui correspond à peu près à la diagnose du genre Favotrichophyton NeveuLemaire 1921 et du genre Grubyella Ota et Langeron, 1923.

Tous ces champignons donnent, sur milieux d'épreuve, des colonies faviformes plus ou moins glabres. L'examen microscopique de ces colonies montre, outre les formes de souffrance qui ont été désignées sous les noms de chandeliers faviques, têtes de clous, etc., des aleuries plus ou moins rudimentaires et quelquefois caduques, des chaìnes de chlamydospores et quelquefois des fuseaux plus ou moins bien différenciés. Tous ces caractères sont communs aux Achorion et aux Trichophyton faviformes. Ces derniers ont d'ailleurs toujours été considérés, notamment par Sabouraud, comme formant un groupe à part parmi les Trichophyton mégasporés.

Les analogies entre Achorion et Trichophyton faviformes se manifestent aussi au cours de l'évolution des lésions. Le godet favique ne se forme pas brusquement, mais d'une manière progressive. On peut le constater en arrachant, sur une tête favique, non seulement les cheveux qui traversent un godet, mais ceux qui paraissent sains ou douteux, et en les examinant au microscope dans le chlorallactophénol. On peut alors observer toutes les formes de transition 
représentées dans la pl. XX. Au début de l'infection, on voit que les Achorion sont mégasporés et endo-ectothrix comme les Trichophyton faviformes. Mais, dans le cas des Achorion, l'organisme humain réagit par une inflammation lente et discrète qui permet l'accumulation des éléments mycéliens et la formation du godet. Au contraire, dans les trichophyties à mégaspores faviformes, chez les animaux ou chez l'homme et notamment chez ce dernier dans certains sycosis, l'organisme réagit par une inflammation plus violente, qui ne permet pas l'accumulation des germes, mais provoque leur expulsion par suite de la suppuration.

Il est difficile de savoir pourquoi, dans un cas, il se forme un godet et dans d'autres cas des lésions suppurées. On ne sait s'il faut attribuer cette propriété aux champignons eux-mêmes ou aux réactions des hôtes parasités. Il semble que c'est plutôt cette dernière influence qui agit, comme il ressort des observations effectuées par l'un de nous au Maroc espagnol. Les teignes y sont très abondantes et il ne semble pas y avoir de relation directe, ni entre le nombre de cas de teignes suppurées des animaux et celui des teignes suppurées à mégaspores faviformes observées chez les enfants, ni entre le nombre de cas de favus infantile et le nombre de cas de favus animai. Par contre, il y a une relation étroite entre les teignes des animaux par mégaspores faviformes et le nombre de cas de favus humain infantile à Achorion.

On peut suivre en quelque sorte expérimentalement l'évolution du favus sur la tête de l'enfant, au moyen de la dépilation par l'acétate de thallium, largement pratiquée par l'un de nous au Maroc. $\mathrm{Si}$ on ne joint pas à cette dépilation un traitement complémentaire, destiné à guérir le favus, on voit ce dernier réapparaitre. On constate qu'il débute toujours par une série de petites pustules périfolliculaires, tout à fait comparables à celles qu'on observe dans les trichophyties à microïdes. On voit les Achorion former des spores ectothrix qui donneront ensuite naissance aux godets par leur accumulation. Ces spores restent autour de l'orifice folliculaire au lieu de former une gaine autour du poil. La lésion initiale du favus paraît donc identique à celle que produisent les mégaspores faviformes, la différence n'est qu'une question de degré dans la réaction inflammatoire. Dans le favus, cette inflammation folliculaire est passagère, très légère et bien tolérée par l'organisme, ce qui permet la formation du godet. Au contraire, dans les trichophyties produites par les mégaspores faviformes, la lésion inflammatoire du follicule est plus intense et moins bien tolérée, ce qui provoque l'expulsion des parasites et empêche la formation des godets.

En ce qui concerne la valeur systématique des six types d'Acho- 
rion que nous décrivons, on peut, suivant qu'on est uniciste ou pluraliste, les considérer soit comme des espèces linnéennes distinctes, soit comme des jordanons formant une grande espèce linnéenne, correspondant au type Achorion schönleini, dont les autres types ne seraient que des sous-espèces ou variétés. L'un de nous a, depuis plusieurs années, introduit, au cours de son enseignement, la notion de jordanon dans l'étude systématique des dermatophytes. Il se produit pour nos nouvelles espèces d'Achorion la même chose que pour les espèces multiples de microïdes (Ctenomyces granulosus, C. asteroides, C. farinulentus, C. radiolatus, C. radians, C. lacticolor). Les différences morphologiques qui séparent ces types de microïdes sont même moins accentuées et moins évidentes que les caractères que nous avons donnés pour nos six Grubyella. Si donc on admet les premières, il n'y a pas de raison pour ne pas accepter les autres. Le cas est encore plus net pour les quelques quinze espèces qui se ramènent toutes au Trichophyton rubrum, comme l'ont bien montré Ota et Kawatsure. La plupart de ces espèces ne sont même pas des jordanons, mais seulement de simples synonymes, qu'aucun caractère sérieux ne permet de séparer du type rubrum.

Quelle que soit la hiérarchie de nos six types d'Achorion, il est certain, d'après nos observations et celles de nos devanciers, que l'Achorion schönleini, produisant la teigne faveuse humaine proprement dite, doit être démembré en plusieurs types spécifiques ou subspécifiques. Tant qu'on ne connaissait que le favus européen, et surtout français, on pouvait admettre qu'il était produit par un parasite monotype, mais l'étude des favus africain et asiatique démontre la multiplicité des parasites du type Achorion.

Les caractères morphologiques que nous venons de décrire et de figurer non seulement confirment les vues de Langeron et Milochevich en ce qui concerne l'étroite parenté des Achorion vrais et des Trichophyton, mais encore nous permettent de faire un pas de plus dans cette voie et de réunir les Achorion vrais aux Trichophyton mégasporés faviformes.

Ces deux types de dermatophytes, tout en restant bien distincts par la nature de la lésion pilaire qu'ils produisent, forment pour nous un groupe pour lequel nous proposons de reprendre la dénomination de Favotrichophyton, créée par Neveu-Lemaire en 1921 et qui a la priorité sur le genre. Grubyella Ota et Langeron 1923.

Ce-groupe serait ainsi constitué :

Groupe Favotrichophyton Neveu-Lemaire 1921.

$1^{\text {re }}$ section. - Favotrichophyton du type Trichophyton endoectothrix mégasporé. Cette section correspond à une partie du genre 
Grubyella Ota et Langeron 1923 ? C'est un groupe très naturel, renfermant les mégaspores des bovins et ovins.
Trichophyton album Sab. 1909.
T. discoides Sab. 1909.
T. ochraceum Sab. 1909.
T. bullosum Lebasque 1933.
T. papillosum Lebasque 1933.
T. pruinosum Catanei 1931 (1).

$2^{\circ}$ section. - Favotrichophyton du type Achorion. Cette section correspond à l'autre partie du genre Grubyella Ota et Langeron 1923. C'est encore un groupe naturel, renfermant les agents du favus humain.
Achorion schönleini (Lebert 1843).
A. pittalugai n. sp.
A. brumpti n. sp.
A. debueni n. sp.
A. talicei, n. sp.
A. milochevitchi $\mathrm{n}$. sp.

A ces deux sections, on peut en joindre une troisième, comprenant des Trichophyton donnant des lésions pilaires purement endothrix, mais formant sur les milieux d'épreuve des colonies faviformes à morphologie microscopique semblable à celle des Achorion.

$3^{\circ}$ section. - Favotrichophyton du type endothrix. Cette section correspond, pour les microspores, au genre Endodermophyton Castellani 1909 et, pour les macrospores, au genre Bodinia Ota et Langeron 1923 (2).

a. - Microspores (anciens Endodermophyton).

Trichophyton concentricum R. Blanchard 1895.

T. roquettei (da Fonseca 1925).

T. langeroni Milochevitch 1931.

b. - Macrospores (anciens Bodinia).

Trichophyton violaceum Bodin 1902.

T. glabrum Sab. 1900.

T. gourvili Catanei 1932.

(1) Les colonies du $T$. pruinosum sur milieux d'épreuve sont entourées d'un cercle de rayons immergés, comme dans notre Achorion milochevitchi. Ces rayons immergés sont encore plus accentués dans un autre Trichophyton faviforme, découvert par S. Milochevitch chez l'homme en Yougoslavie, mais donnant une lésion purement endothrix à très petites spores. Ce Trichophyton a reçu le nom de $T$. langeroni Milochevitch 1931 .

(2) On sait, depuis les recherches de Langeron et Milochevitch (1930), que les genres Endodermophyton et Bodinia n'ont plus de raison d'être en tant qu'unités systématiques. 
Bien que voisins par l'aspect macroscopique et microscopique de leurs colonies sur milieux d'épreuve, les dermatophytes qui forment ces trois sections diffèrent nettement par la nature des lésions qu'ils produisent. Comme la classification des dermatophytes repose en grande partie sur la morphologie microscopique des lésions pilaires, telle que Sabouraud l'a établie, nous croyons préférable de ne pas réunir dans un seul genre des champignons dont les propriétés biologiques sont si différentes et de n'attribuer au terme Favotrichophyton que la valeur d'un groupement commode pour faire ressortir la propriété commune à tous ces champignons de donner des colonies glabres sur milieux d'épreuve.

\section{BiBLIOGRAPHIE}

Bazza (M.). - Aportacion al estudio de las tiñas del Norte de Marruecos. Comision permanente de investigaciones sanitarias, Madrid, $1934,15 \mathrm{p}$. Note statistique préliminaire sur les teignes du Maroc espagnoi. Ann. de Parasitol., XII, 1934, p. 405-407.

- Observaciones sobre la biologia de los penicilios, etc. Comision permanente de investigaciones sanitarias, Madrid, 1934, 26 p.

BLocн (B.). - Der Achorion violaceum ein bisher unbekannter Favuspilz. Dermat. Ztschr., 1911, p. 815.

Bodis. - Sur un nouveau champignon du favus (Achorion gypsenm). Ann. de Dermat. et Syphil., 1907, p. 585.

Cataxer. - Sur la production d'appareils sporifères par un champignon du groupe des Trichophyton à culture faviforme. C.R. Soc. Biol., CV, 1930 , p. 504 .

\section{EXPLICATION DES PLANCHES XV-XX}

\section{Planche XV}

FIG. 1. - Achorion schönleini : aspect d'un cheveu favique dans le chlorallactophénol (préparation récente).

Fig. 2. - Type pittalugai : aspect macroscopique des colonies sur milieux d'épreuve glycosé, maltosé et polysucré (colonies âgées de 28 jours).

Fıg. 3. - Achorion schönleini : autre aspect d'un cheveu favique dans le chloral-lactephénol (préparation ancienne et chauffée; l’air a été expulsé, ce qui permet de voir les filaments mycéliens qui remplissent le cheveu.

FıG. 4. - Achorion schönleini : aspect macroscopique des colonies en tube sur milieu d'épreuve de Sabouraud polysucré.

Fig. 5. - Type pittalugai : aspect microscopique du cheveu favique dans le chloral-lactophénol. 
Chen, Kunotchкin and Hu. - Tinea favosa in Peiping. National. Med. Jour. of China, XVII, 1931, p. 529-533.

Dey (N. C.) et Maplestone (P. A.). - Favus in India, Indian journ. med. res., XXIII, 1936, p. 687-699.

Grigonaki. - Sur une nouvelle espèce de favus: Arthrosporia gougeroti. C.R. Soc. Biol., CXIV, 1933, p. 258.

Hashimoto, Ishibashi, Iwatake et Ota. - Le favus en Mongolie et son champignon causal : Grubyella schönleini var. mongolica. Japan, Journ. Derm. Urol., XXVII, $1927, \mathrm{n}^{\circ} 5$.

Langeron et MiLochevitch. - Morphologie des dermatophytes sur milieux naturels et milieux à base de polysaccharides. Essai de classification. Ann. de Parasitol., VIII, 1930 , p. 465-508.

Mrochevitch. - Un cas de favus du cuir chevelu produit par le Trichophyton faviforme album. Medicinski Pregled, sept. 1933.

Ota (M.) et Langeron (M.). - Nouvelle classification des dermatophytes. Ann. de paras., I, 1923, p. 305.

Neveu-Lemaire (M.). - Précis de parasitologie humaine, 5e édition. Paris, Lamarre, 1921.

Sabouraud et Negroni. - Aleuries et rudiments de fuseaux obtenus sur la culture d'Achorion schönleini. Ann. Dermat. et Syphil. (6), X, 1929, p. 232-235.

\section{Section de Mycologie de l'Institut de Parasitologie}

de la Faculté de Médecine de Paris (Chef de service: $D^{\mathrm{r}}$ M. Langeron)

Fıg. 6. - Achorion schönleint : dessin réunissant les divers éléments morphologiques du champignon : A, aleuries ; $\mathrm{N}$, organe nodulaire ; $\mathrm{C}$, chlamydospores; E, chlamydospores en chaîne; F, chandeliers faviques.

Fıg. 7. - Achorion schönleini : microphotographie d'une culture sur lame, montrant la formation des aleuries (sans fixation, coloration au bleu coton à 1 p. 1.000 dans le lactophénol).

\section{Planche XVI}

Fig. 8. - Type pittalugai : dessin réunissant les divers éléments morphologiques observés dans les cultures sur lame: A, aleuries ; C, chlamydospores; $c h$, chlamydospores prenant l'aspect de fuseaux ; E, chlamydospores en chaîne; $\mathrm{F}$, chandeliers faviques ; $\mathrm{P}$, organes pectinés.

Fig. 9. - Typé brumpâi : dessin réunissant les éléments morphologiques d'une culture sur lame: A, aleuries; C, chlamydospores; E, chlamydospores en chaînes ; $\mathrm{N}$, organe nodulaire.

F16. 10. - Type pittalugai : microphotographie montrant les aleuries (A) (culture sur lame, coloration au bleu coton à 1 p. 1.000 dans le lactophénol).

Fig. 11. - Type brumpti : aspect microscopique du cheveu favique dans le chloral-lactophénol.

Fic. 12. - Type brumpti : aspect des colonies primaires sur milieu d'épreuve glycosé.

FIG. 13. - Type brumpti : aspect des colonies secondaires sur milieux d'épreuve glycosé, maltosé et polysucré.

Annales de Parasitologie, T. XIV, $\mathrm{N}^{\circ} 4 .-1^{\mathrm{er}}$ juillet 1936. 


\section{Planche XVII}

FIG. 14. - Type brumpti : microphotographie d'une culture sur lame en milieu sucré, fixée et colorée au Ziehl.

FIG. 15. - Type brumpti : microphotographie d'une autre culture sur lame sur milieu polysucré, montrant les aleuries (préparation non fixée et colorée au bleu coton, au lactophénol à 1 p. 1.000).

Fig. 16. - Type debueni : dessin réunissant les éléments d'une culture sur lame sur milieu polysucré : A, aleuries; C, chlamydospores ; N, organes nodulaires ; F, chandelier favique ; H, fuseau ; E, chlamydospores en chaine.

Fig. 17. - Type debueni : microphotographie d'une culture sur lame en milieu polysucré (préparation non fixée, colorée au bleu coton à 1 p. 1.000 dans le lactophẻnol).

\section{Planche XVIII}

Fig. 18, 19, 20. - Type debueni : aspect des colonies sur milieux d'épreuve glycosé, maltosé et polysucré (cultures âgées d'un mois).

Fig. 21. - Type talicei: aspect des colonies sur milieux d'épreuve glycosé, maltosé et polysucré (cultures de 28 jours).

Fig. 22: - Type debueni : aspect microscopique d'un cheveu favique, dans le chloral-lactophénol.

Fig. 23. - Type tažicei : aspect microscopique d'un cheveu favique dans le chloral-lactophénol.

Fig. 24. - Type talicei : dessin réunissant les éléments d'une culture sur lame en milieu polysucré.

Fig. 25. - Type talicei : microphotographie d'une culture sur lame, montrant les fuseaux $(\mathrm{H})$.

Fig. 26. - Type talicei : microphotographie d'une culture sur lame, montrant les alcuries et les fuseaux (préparation non fixée, colorée au bleu coton à 1 p. 1.000 dans le lactophénol).

\section{Planche XIX}

Fig. 27, - Type milochevitchi : aspect des colonies sur milieux d'épreuve glycosé, maltosé et polysucré (culture de 28 jours).

Fig. 28. - Type milochevitchi : aspect microscopique du cheveu favique dans le chloral-lactophénol.

Fig. 29. - Type milochevitchi : microphotographie d'une culture sur lame en milieu polysucré (préparation non fixée, colorée au bleu coton à 1 p. 1.000 dans le lactophénol).

Fig. 30. - Type milochevitchi: dessin réunissant les éléments d'une culture sur lame en milieu polysucré.

Fig. 31. - Type milochevitchi : microphotographie d'une culture sur lame en milieu polysucré (préparation non fixée, colorée au bleu coton à 1 p. 1.000 dans le lactophénol).

\section{Planche XX}

FıG. 32. - Achorion schönleini : aspect d'un cheveu favique dans le chlorallactophénol.

Fig. 33. - De gauche à droite: souches 706 (souche Milochevitch $\mathrm{n}^{\circ}$ 38, de Belgrade), 529 (souche algérienne, El Kantara 1, de l'Institut Pasteur d'Algérie), 376 (souche du $\mathrm{D}^{\mathrm{r}}$ Smith, de Lagos, Nigeria).

Fig. 34, 35, 36. - Mode de formation du godet favique. Etapes successives. 
ANNALES DE PARASITOLOGIE

T. XIV, No 4, 1er Juillet 1936

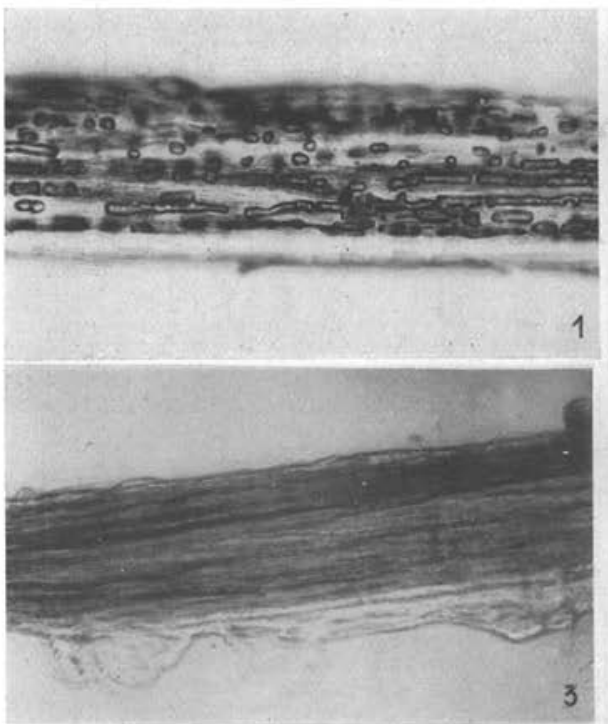

Planche XV

(Mémoire Langeron et BaEza)
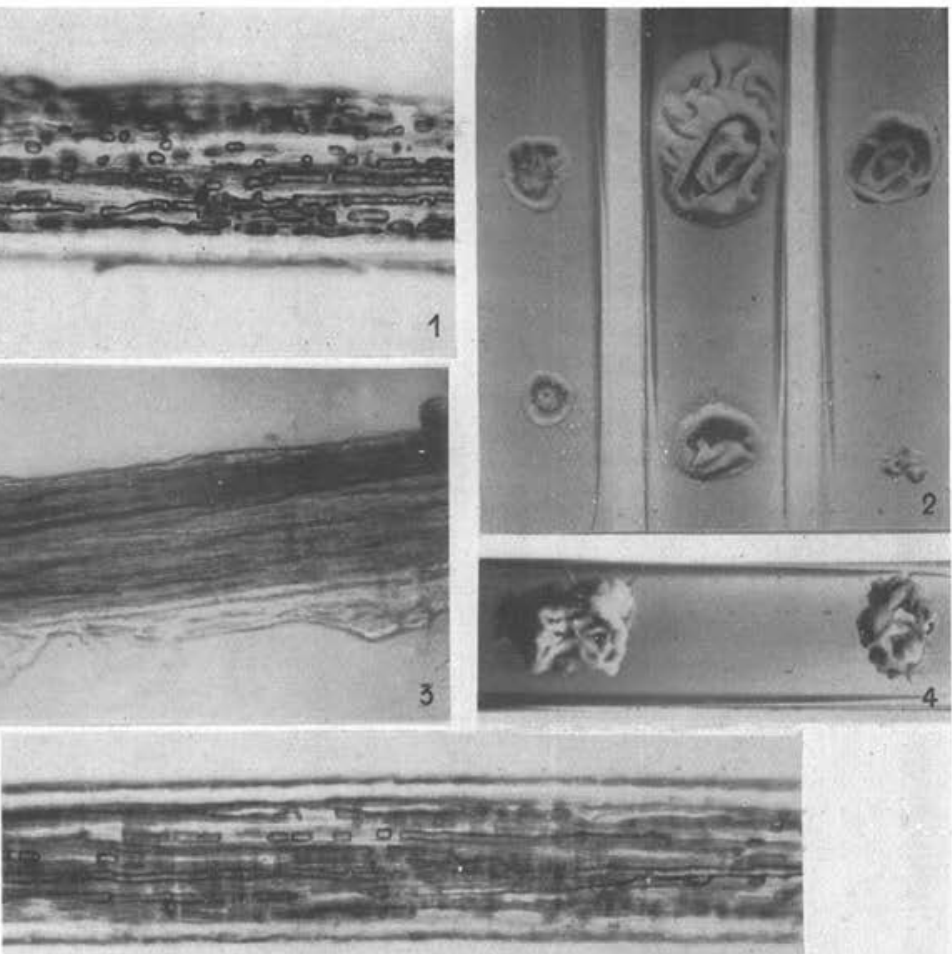

5
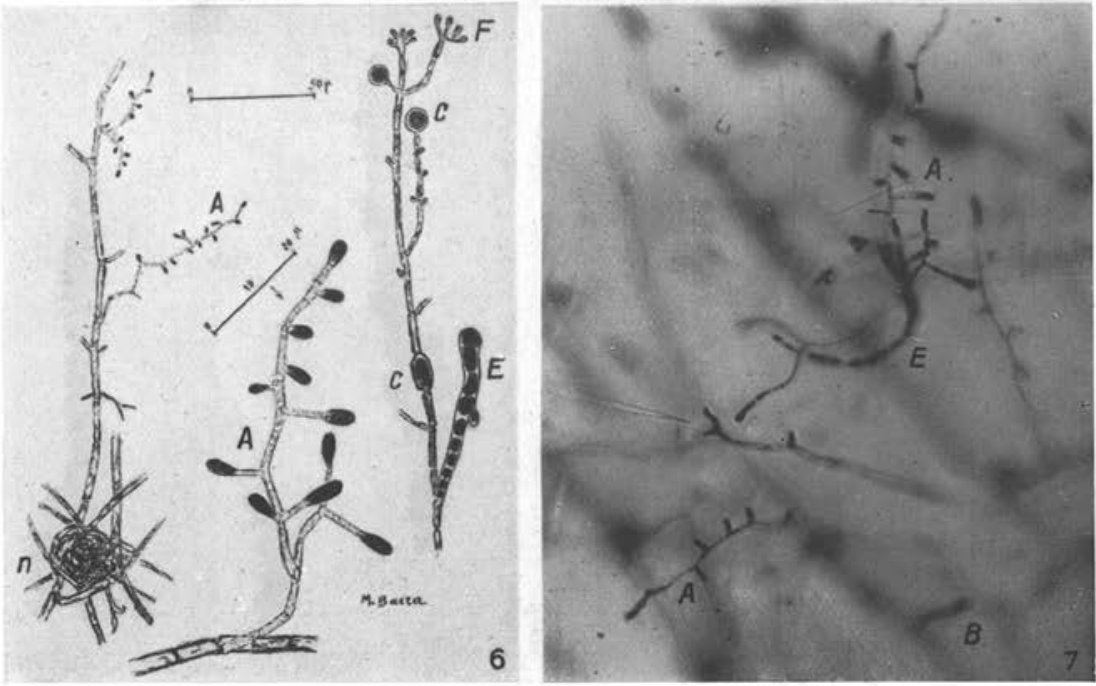

Masson et Cie, Emiteuns 

IALES DE PARASITOLOGIE

. XIV, No 4, 1er Juillet 1936
Planche XVI

(Mémoire Langeron et BaEza)
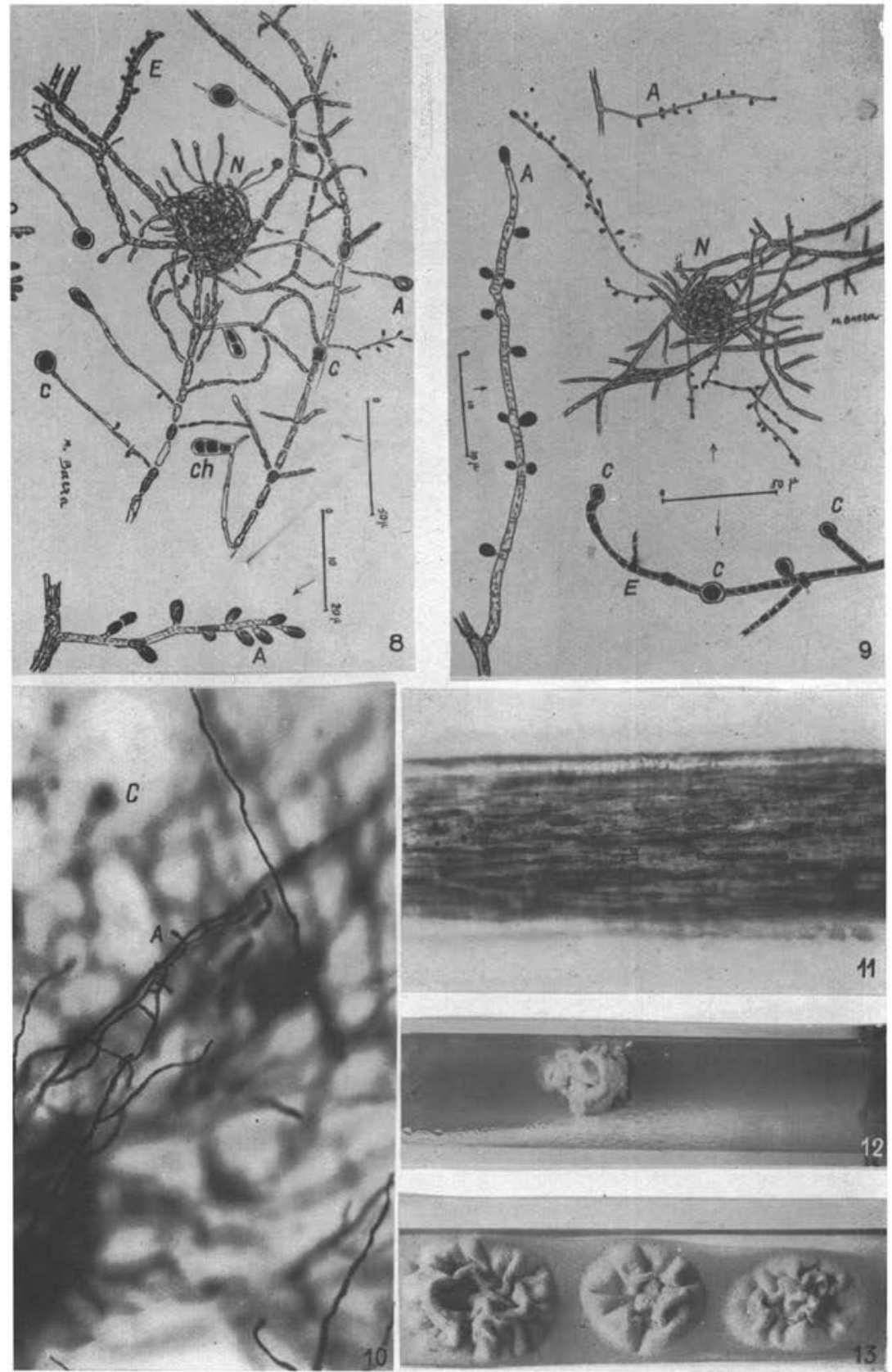
ANNALES DE PARASITOLOGIE

T. XIV, No 4, 1er Juillet 1936
Planche XVII

(Mémoire Langeron et BaEza)
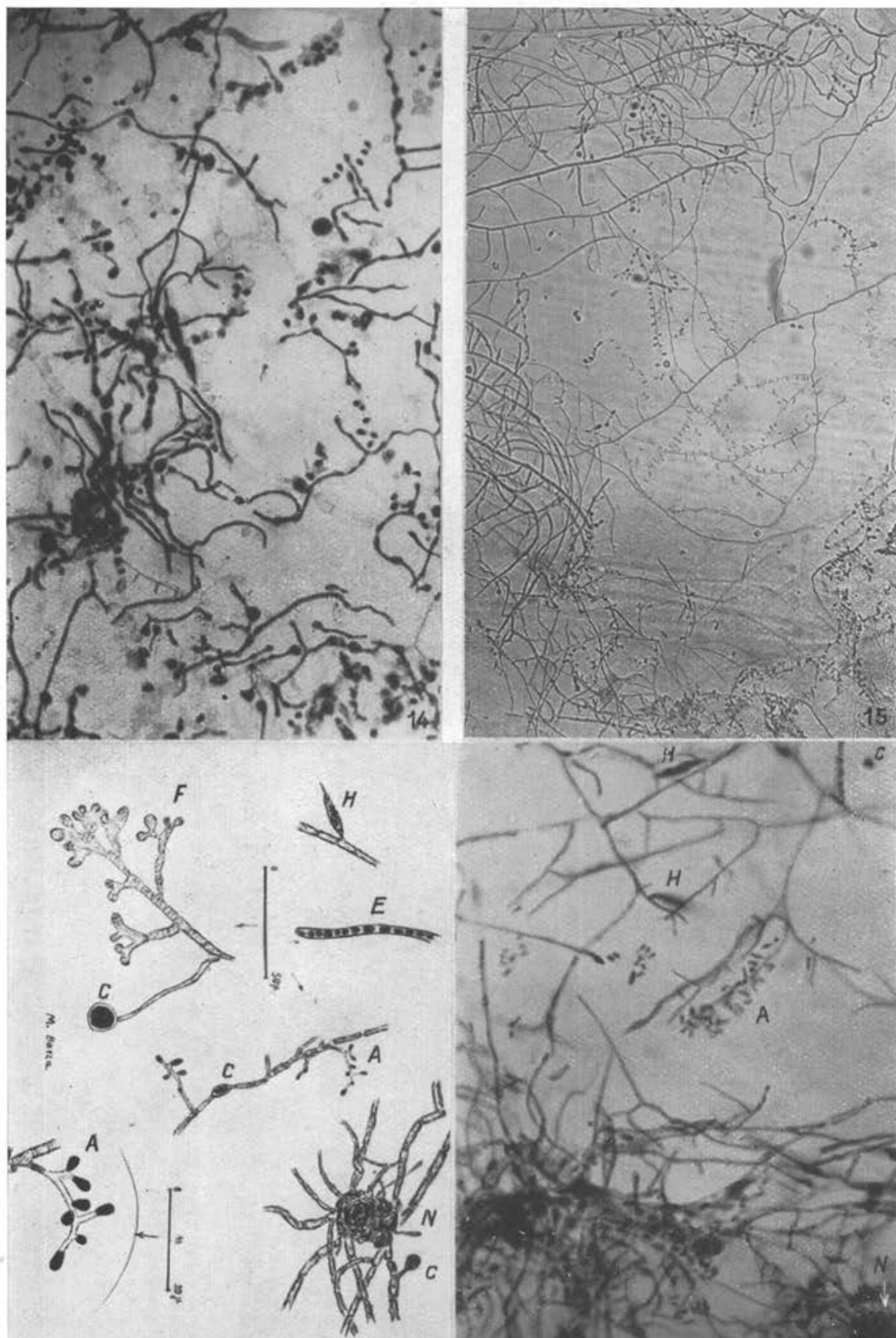

If

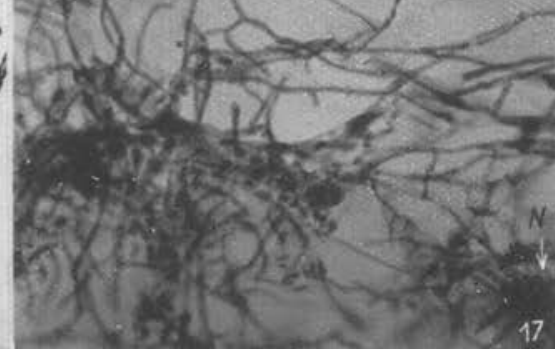



ANNALES DE PARASITOLOGIE

T. XIV, No 4, 1er Juillet 1936
Planche XVIII

(Mémoire Langeron et BaEza)
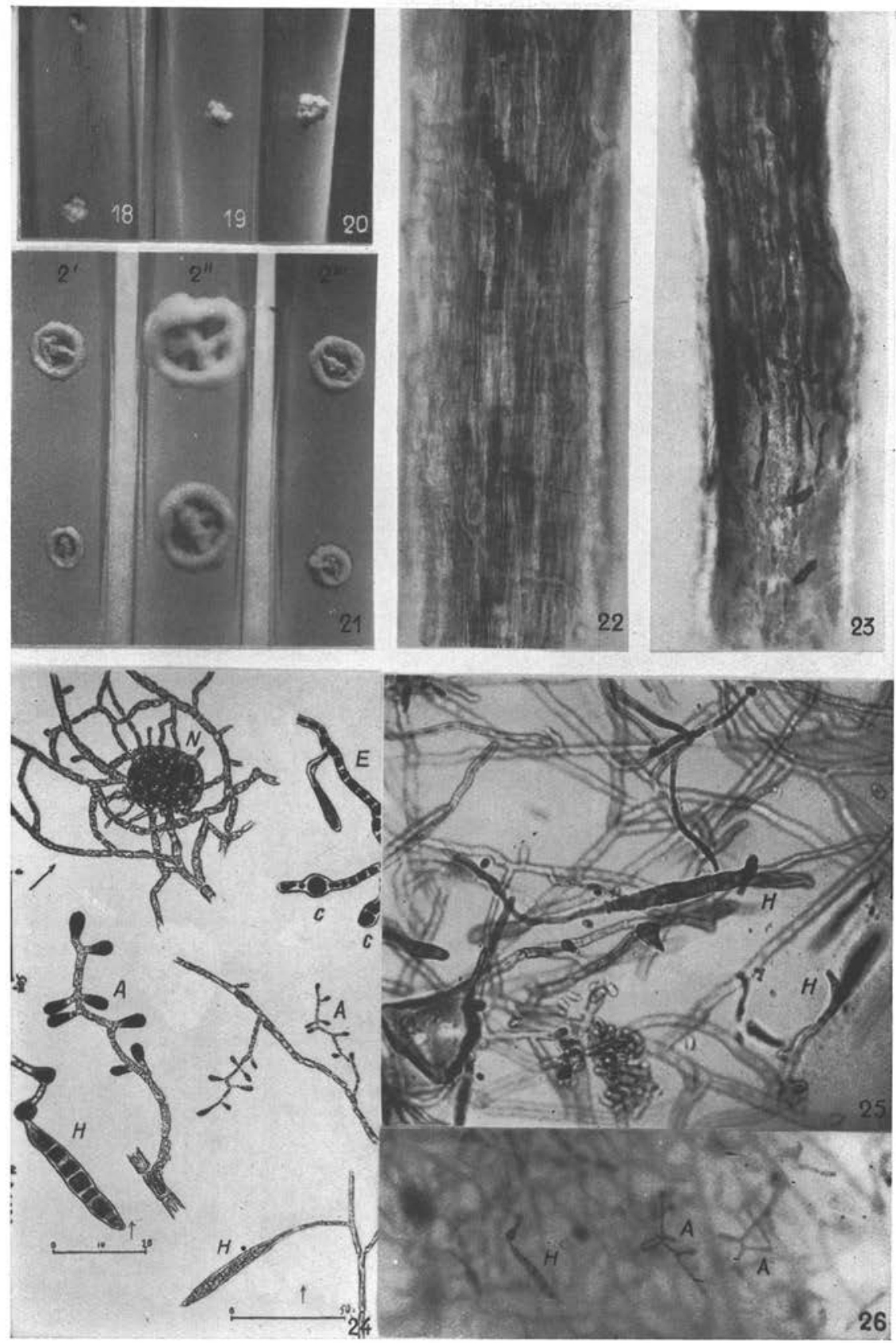
ANNALES DE PARASITOLOGIE

T. XIV, No 4, 1er Juillet 1936
Planche XIX

(Mémoire Langeron et B.aza)

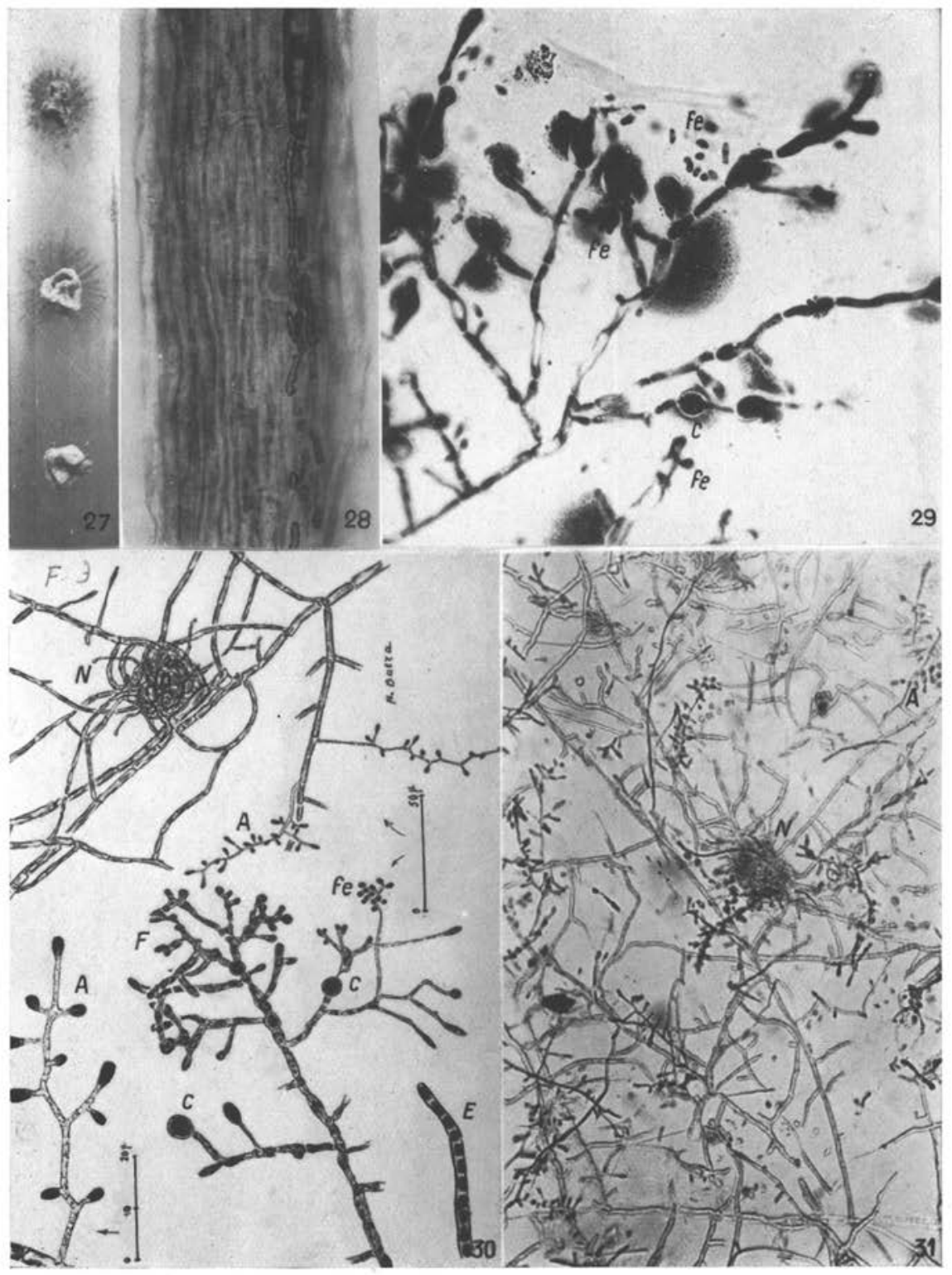



ANNALES DE PARASITOLOGIE

T. XIV, No 4, 1er Juillet 1936
Planche XX

(Mémoire Langeron et Bafza)
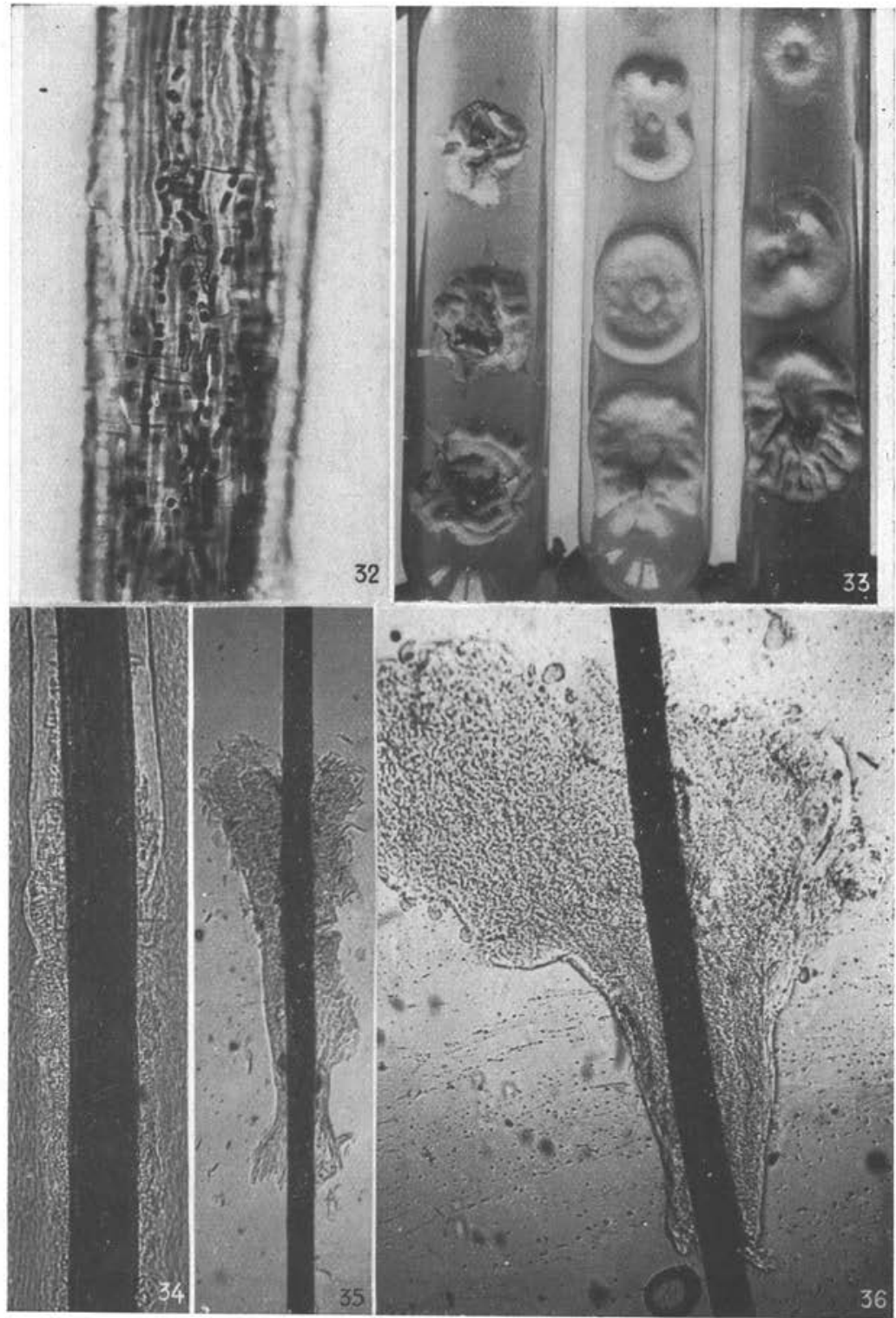